\title{
FLOW MODELING IN AN EVAPORATOR OF A NONCONVENTIONAL ENERGETIC SYSTEM
}

An alternative cooling medium (lithium bromide liquid) flows in a cooling jacket of a combustion engine of a nonconventional energetic unit. The paper deals with a flow simulation of the working medium in the vaporization container of a cooling circuit of the nonconventional combustion engine. Consequently evaporation and thermal states at the atmospheric pressure above the nonconventional cooling liquid (solution $\mathrm{LiBr}-\mathrm{H} 2 \mathrm{O}$ ) level in the evaporator are simulated. Limiting conditions for the functionality assurance of the cooling system of the nonconventional combustion engine were found by means of a simulation.

\section{Introduction}

A nonconventional cooling-combustion engine uses the part of energy bound in a cooling system of a combustion engine for production of the cooling output applicable in vehicles (see Fig. 1) [2].

An engine cooling liquid is replaced by mixture of the lithium bromide liquid with water [6]. The solved energetic system is defined in area of trigeneration systems. The mentioned system is a source of electrical energy, cold and heat. The engine used in the system is four stroke compression ignition engine Z8004 with rated power of $77 \mathrm{~kW}$ at $2200 \mathrm{~min}^{-1}$. The heat produced by the engine cooling system is pulled into the absorption cooling equipment.

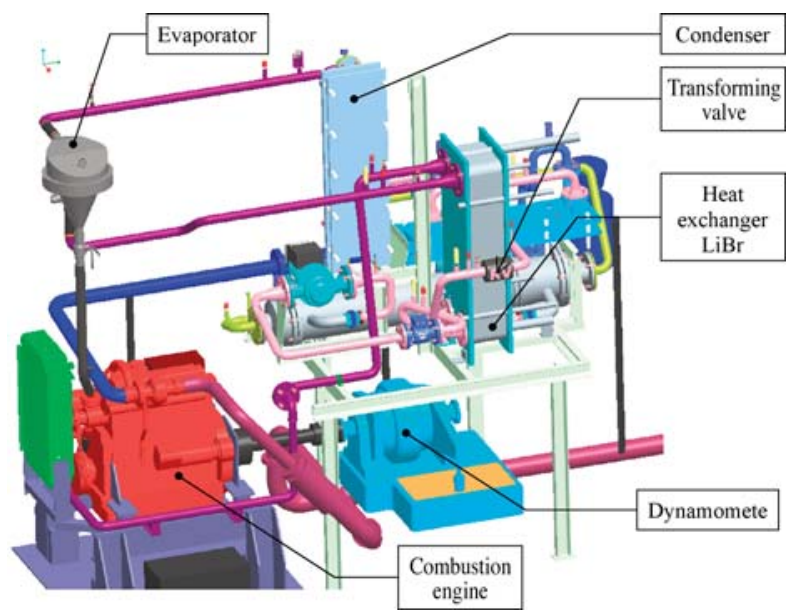

Fig. 1 Virtual model of the non-conventional energetic unit [2]
The article shows the flow simulation of the working medium in this structural shape of vaporization container of a cooling circuit of the nonconventional combustion engine. The problem with vaporization container was that the off-take wasn't able to take away the entire needed amount from the evaporator.

\section{Definition of the model and the flow simulation}

The CAD software used for the model creation is CATIA V5 [2]. The model of the whole evaporator can be seen in Fig. 2.

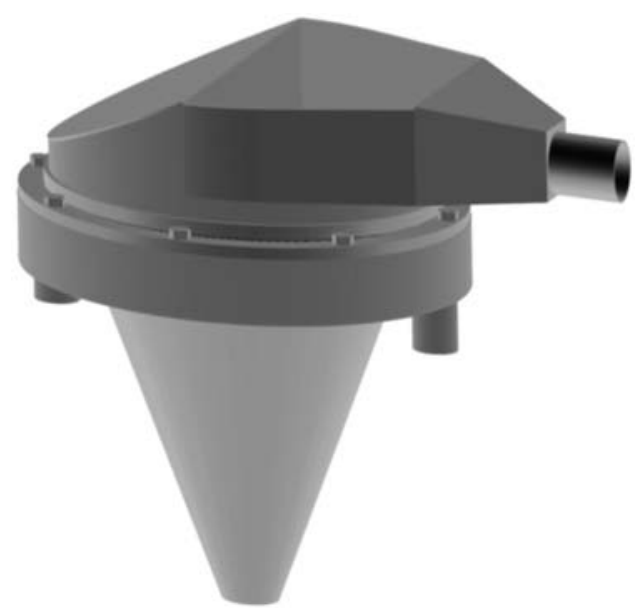

Fig. 2 CAD model of the evaporator

For the purpose of simulation we used only one half of this space because of the symmetry of the geometry which allows for

\footnotetext{
* Andrej Kovalcik, Emil Toporcer, Vladimir Hlavna

Faculty of Mechanical Engineering, University of Zilina, Slovakia, E-mail: andrej.kovalcik@fstroj.uniza.sk
} 
decrease in the requirements for the used hardware and computational time.

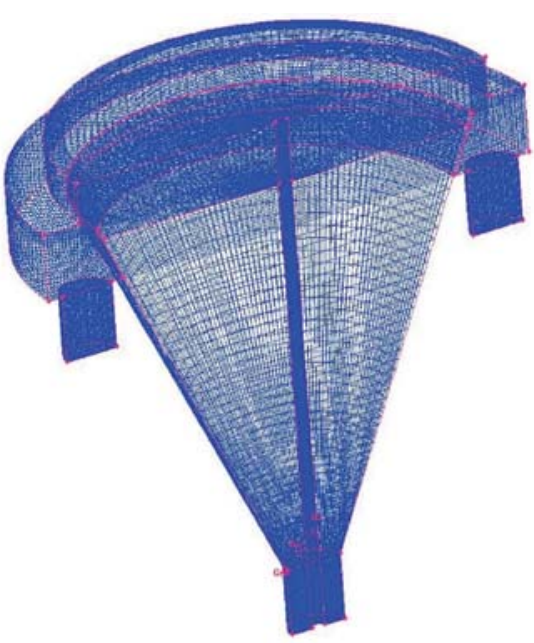

Fig. 3 View of the model of evaporator [5]

Fig. 3 shows the mesh in the area of the bottom outlet. Then the suitable types of boundary conditions are applied to the mesh model (see Fig. 4). The resulting mesh contains about 480000 elements [5].
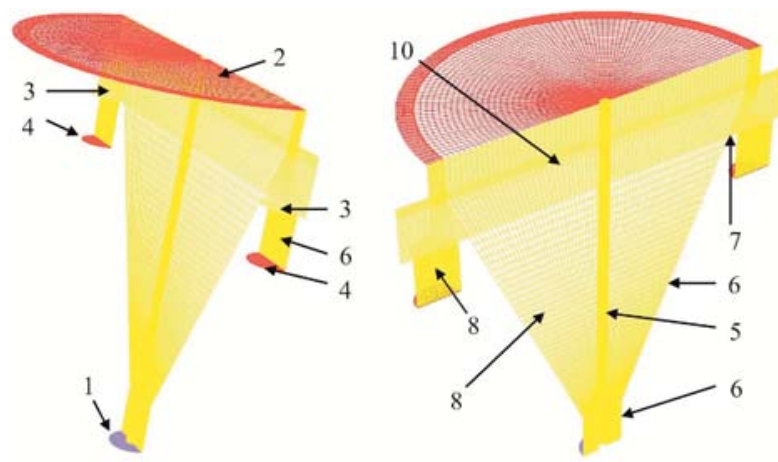

1-velocity inlet I, 2-pressure outlet I, 3-velocity inlet II, 4-pressure outlet II, 5-axis, 6-wall, 7-interface wall + velocity inlet II, 8-symetry

Fig. 4 Boundary conditions

Next step was the definition of the computational model. Values for the boundary conditions were set. The RNG $k-\varepsilon$ turbulent model was used for the flow simulation. This model is robust enough and suitable for the solution of turbulent flows and heat transfer [4]. The renormalized procedure consists of a gradual elimination of small turbulences. Equations of motion are transformed so that turbulent viscosity, forces and nonlinear members are modified. Assuming that the turbulences are related to dissipation $\varepsilon$, then, the turbulent viscosity $\mu$, depends on a degree of turbulences and the RNG method constructs this viscosity with the help of iterative elimination of narrow bands of wave numbers. The following equation is used for the iterative process [ 1 and 5]:

$$
\frac{d \mu_{e f f}}{d l}=\frac{A_{l} \varepsilon l^{3}}{\mu(l)^{2}}
$$

The RNG model derived by a statistical method, averaged, is formally of the same form as the classical $k-\varepsilon$ model. The equation for the transfer of motion is in the form:

$$
\begin{aligned}
& \frac{\partial}{\partial t}\left(\rho \bar{u}_{i}\right)+\frac{\partial}{\partial x_{j}}\left(\rho \bar{u}_{i} \bar{u}_{j}\right)= \\
& =\frac{\partial}{\partial x_{j}}\left[u_{e f f}\left(\frac{\partial \bar{u}_{i}}{\partial x_{j}}+\frac{\partial \bar{u}_{j}}{\partial x_{i}}\right)-\left(\frac{2}{3} u_{e f f} \frac{\partial \bar{u}_{i}}{\partial x_{i}}\right)\right]-\frac{\partial \bar{p}}{\partial x_{i}}+\rho g_{i}+F_{i}
\end{aligned}
$$

and, subsequently, transport equations are used:

$$
\begin{aligned}
& \frac{\partial}{\partial t}(\rho k)+\frac{\partial}{\partial x_{j}}\left(\rho \pi_{j} k\right)= \\
& =\frac{\partial}{\partial x_{j}}\left(\alpha_{k} \mu_{e f f} \frac{\partial k}{\partial x_{j}}\right)+\mu_{t} S^{2}-\rho \varepsilon
\end{aligned}
$$

$$
\begin{aligned}
& \frac{\partial}{\partial t}(\rho \varepsilon)+\frac{\partial}{\partial x_{j}}\left(\rho \mu_{j} \varepsilon\right)= \\
& =\frac{\partial}{\partial x_{j}}\left(\alpha_{\varepsilon} \mu_{e f f} \frac{\partial \varepsilon}{\partial x_{j}}\right)+C_{1 \varepsilon} \frac{\varepsilon}{k} \mu_{t} S^{2}-C_{2 \varepsilon} \rho \frac{\varepsilon^{2}}{k}-R
\end{aligned}
$$

Time step was set to $0.001 \mathrm{~s}$. The velocity in the inlet was set to $0.68 \mathrm{~m} \cdot \mathrm{s}^{-1}$. Fig. 5 shows the level of the cooling liquid at the start of the simulation process $(1 \mathrm{~cm}$ under the edge of the evaporator cone).
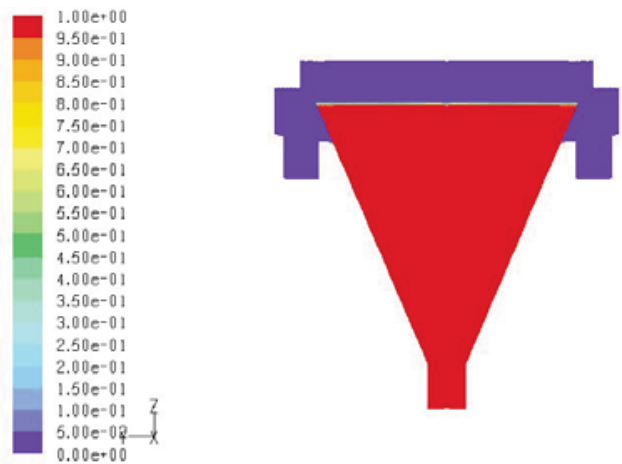

Contours of Volume fraction (libr) (Time- 0.0000 e +00 ) Jan 13. 2010

Fig. 5 Coolant level in the evaporator at the start of the simulation

\section{Achieved results}

The filling of the evaporator ring by the cooling medium is shown in Figs. 6, 7, 8 and 9. The off-take is able to take away the 
entire needed amount from the evaporator. The simulation shows that the lithium bromide liquid starts to pour through the edge of the evaporator cone in the time of about $0.96 \mathrm{~s}$ after the initiation of the calculation. The starting cooling liquid level has the value of $1 \mathrm{~cm}$ under the edge of the evaporator cone. The value of $1 \mathrm{~cm}$ is used because of the decrease in the computational time.

The found value of $0.96 \mathrm{~s}$ valid for the $55 \%$ lithium bromide liquid cannot be experimentally verified by means of simulation due

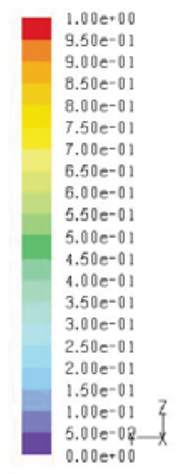

$\begin{aligned} & \text { Contours of Volume fraction (libr) (Time-1.0000e }+00 \text { ) } \\ & \text { FLUENT } 6.3 \text { (3d. pbns, vof, ingke. unsteady) }\end{aligned}$

Fig. 6 Flow in the evaporator in the time of $1 \mathrm{~s}$

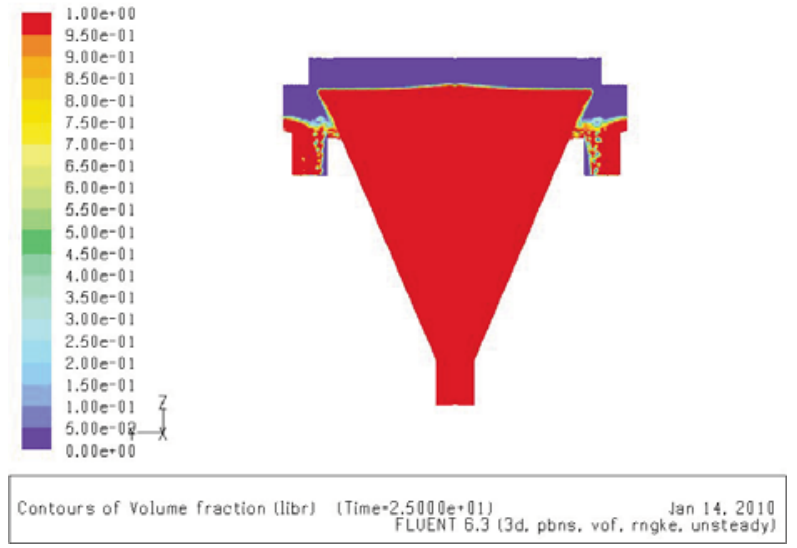

Fig. 8 Flow in the evaporator in the time of $25 \mathrm{~s}$

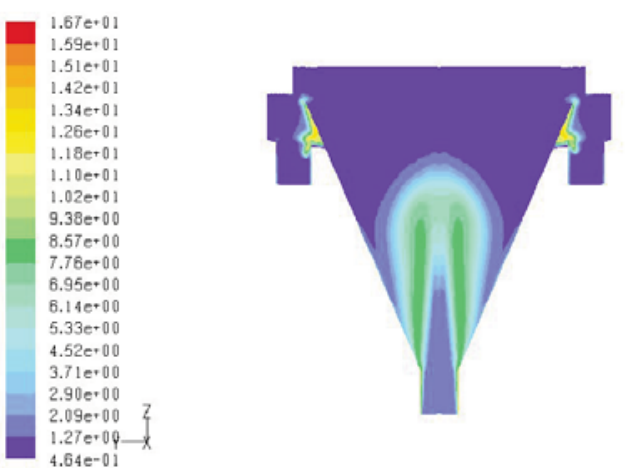

Contours of Turbulent Intensity (mixture) (I) (Time-1.0000e.00) Jan 13.2010
FLUENT 6.3(3d. dp. pbns. vof, mgke. unsteady)

Fig. 10 Turbulent intensity in the time of $1 \mathrm{~s}$

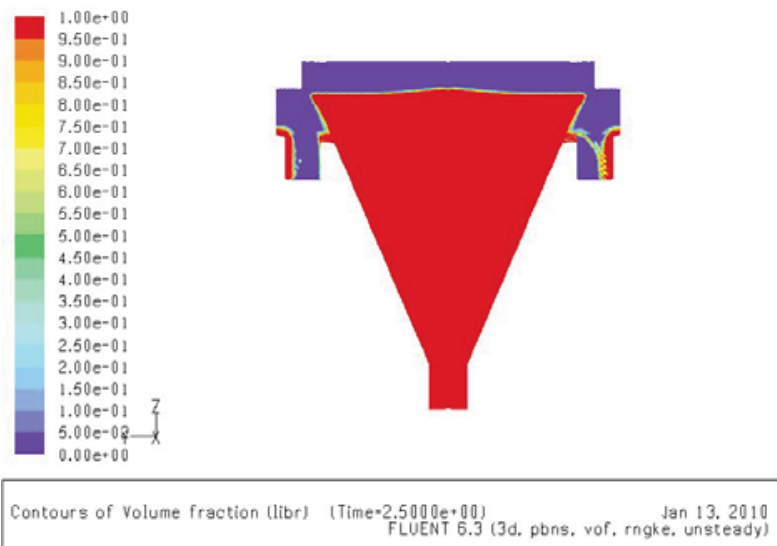

Fig. 7 Flow in the evaporator in the time of $2.5 \mathrm{~s}$
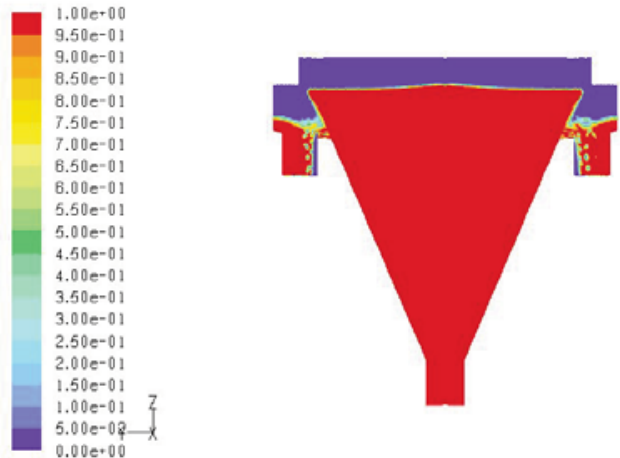

Contours of Volume fraction (libr) (Time-3.5000e 01 )
FLUENT 6.3 (3d. pbns, vof, make. Unsteady)

Fig. 9 Flow in the evaporator in the time of $35 \mathrm{~s}$
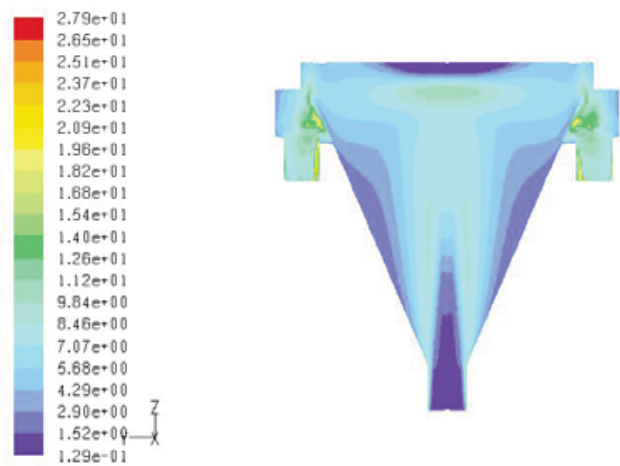

Contours of Turbulent Intensity (mixture) (\$) (Time-2.5000e.01) Jan 13.2010

Fig. 11 Turbulent intensity in the time of $25 \mathrm{~s}$

10 COMMUNICATIONS 4/2011 
to the fact that water is at present time the coolant in the cooling circuit of the engine.

Figs. 10 and 11 illustrate that the maximal values of turbulence intensity are situated in the area of cylindrical walls of the inflow and outflow pipes, edge of the evaporator cone and at the lithium bromide liquid level in the centre of the evaporator [5].

\section{Conclusion}

The simulation shows that the cooling medium starts to pour through the edge of the evaporator cone in the time of about $0.96 \mathrm{~s}$ after the simulation starts. The inflow and outflow pipes, edge of the evaporator cone and the area of the coolant level in the evaporator centre are the places of the turbulent intensity maximal values.

\section{Acknowledgement:}

The contribution was created within the framework of the projects APVT-20-018404 and SK-PL-0035-09, which are supported by the Agency for Support of Science and Technology of the Slovak Republic.

\section{References}

[1] ElSAFTY, A., AI-DAINI, A. J.: A Parametric Study of Vapor Absorption Air-conditioning System Soe, Coventry University, Coventry, 2003.

[2] HLAVNA, V., SOJCAK, D.: An Analysis of Heat Flows in the Combustion Engine of a Ship Non-conventional Energetic System, Communications - Scientific Letters of the University of Zilina, 1/2005, ISSN 1335-4205.

[3] CHALAMONSKI, M.: Analysis of Thermal Centre Failuring, Eksploatacja i niezawodnosc, Nr. 4(36)/2007, ISSN 1507-2711.

[4] KOVACIK, O., ORSANSKY, P.: Partial Differential Equation for Heat Conduction and its Solvability, Communications - Scientific Letters of the University of Zilina, No. 1, 2010, ISSN 1335-4205.

[5] KOVALCIK, A.: Cooling Combustion Engine as a Non-conventional Energetic Unit, SjF, University of Zilina, 2010.

[6] SENCAN A., YAKUT, A., DIKMEN, E.: New Model for Determining the Thermodynamic Properties of libr-h2o Solution, G. $U$. J. of Science 17(1): 101-110-2004, ISSN 1303-9709. 